\title{
Qingfei Xiaoyan Wan alleviates asthma through multi-target network regulation
}

\author{
Zhenying Zhao ${ }^{1,2}$, Yingbo Miao ${ }^{1}$, Pengwei Pan ${ }^{1}$, Binfeng Cheng ${ }^{1}$, Gang Bai ${ }^{1}$ and Hong $\mathrm{Wu}^{3^{*}}$
}

\begin{abstract}
Background: Qingfei Xiaoyan Wan (QFXY), a traditional Chinese formula, is widely used for relieving cough, asthma, upper respiratory tract infection, bronchitis, pneumonia, and etc. in clinic. Comparing with other anti-asthma drugs, it is characterised with moderate and persistent efficacy as well as few side effects, however, the underlying action mechanism still remains elusive. This study aimed to identify QFXY multi-target network regulation as an asthma controller.
\end{abstract}

Methods: This study established asthma model induced by histamine phosphate and acetylcholine chloride (His\&Ach) in guinea pigs, which then were administered orally with QFXY. Hematoxylin-Eosin staining sections were applied for evaluating QFXY effect. In both Model and QFXY groups, customized microarrays and 2D electrophoresis were adopted to detect differentially expressed genes (diff genes) and proteins (diff proteins) respectively, and some diff proteins were identified with MALDI-TOF/MS. The checked diff genes and proteins underwent Cluster, GO and KEGG analysis. Based on GAD and HPRD databases, QFXY-asthma target regulation network was constructed.

Results: His\&Ach-induced asthma model of guinea pigs was established. HE sections presented anti-inflammation and anti-remodelling effects of QFXY. Comparing with the Model group, 55 diff genes and 6 diff proteins were identified in QFXY group. Validation by qPCR and Western blot showed the microarray and 2D data reliable. Furthermore, QFXY-asthma target regulation network was achieved.

Conclusions: A primarily combined genomic and proteomic screening of QFXY targets displayed a series of candidate genes and proteins, which indicated that the effect of QFXY relied on the combined mechanism, anti-inflammation and anti-remodelling, as well as influencing signal transduction in vivo.

Keywords: Asthma, Traditional Chinese Medicine, Microarray, 2D- electrophoresis, MS identification, Multi-target network

\section{Background}

Asthma, as defined in 2008 by the Global Initiative for Asthma (GINA), is an inflammatory disorder of the airways in which many cells and cellular elements play roles [1]. Bronchial hyperactivity associates with inflammation, that together with an external or environmental insult, on a vulnerable bronchial epithelial structures, generates tissue remodelling and respiratory functional impairment [2]. Asthma is not a curable disease at the present time [3,4]. However, with proper treatments, the risk of mortality for asthmatic individuals could be

\footnotetext{
* Correspondence: wuhongmedu@126.com

${ }^{3}$ Department of Pharmacology, Tianjin Medical University, Tianjin, People's Republic of China

Full list of author information is available at the end of the article
}

comparable to that of the general population. Presently, the treatment of asthma includes a dual focus: the shortterm treatment of acute symptoms with bronchodilators, and together with the prevention or eventual reversal of chronic inflammation using anti-inflammatory drugs [5].

Medications to treat asthma can be classified as controllers or relievers. Controllers are medications taken daily on a longterm basis to keep asthma under clinical control chiefly through their anti-inflammatory effects. Relievers are medications used on an as-needed basis, which act quickly to reverse bronchoconstriction and relieve its symptoms $[1,6]$. The major medications in asthma management include bronchodilator $\beta_{2}$-agonists, anti-inflammation inhaled corticosteroids, leukotriene 
modifiers and theophyllines. The use of rapid-acting $\beta_{2^{-}}$ agonists in long period may lead to relative refractoriness to $\beta_{2}$-agonists [7]. Long-acting inhaled $\beta_{2}$-agonists, including formoterol and salmeterol, should never be used as monotherapy for asthma as these medications do not appear to influence the airway inflammation in asthma. They are most effective when combined with inhaled glucocorticosteroids, and this combination therapy is the preferred treatment when a medium dose of inhaled glucocorticosteroid alone fails to achieve control of asthma [8,9]. Inhaled glucocorticosteroids are currently the most effective anti-inflammatory medications for the treatment of persistent asthma. The systemic side-effects of long-term treatment with high doses of inhaled glucocorticosteroids include easy bruising, adrenal suppression and decreased bone mineral density and etc. When the drugs are discontinued, deterioration comes out within weeks to months in proportion of cases [1]. Leukotriene modifiers are associated with dose reductions of inhaled glucocorticosteroids, while monitoring of liver tests is recommended during their treatment for the underlying liver toxicity [10]. Theophylline, a bronchodilator, when given in a lower dose, has modest anti-inflammatory properties, but needs proper monitoring for its narrow therapeutic range [11]. As men tioned above, there is a consistent need to explore novel effective anti-inflammation and bronchodilator drugs, especially suitable for the senior and children or chronic patients.

QFXY is originated from a famous Traditional Chinese Medicine (TCM) formula "Maxing Shigan Decoction". It has been experimentally improved, consisting of eight materia medicas, Ephedra Herba, Saigae Tataricae Cornu, Pheretima, Arctii.

Fructus, Lepidii Semen, Bovis Calculus Artifactus, Arme niacae Semen Amarum and Gypsum Fibrosum. Since decades of extensive clinical practice, QFXY has shown significantly therapeutic effects on dissolving phlegm as well as relieving cough, asthma, upper respiratory tract infection, bronchitis, pneumonia, and etc., but its underlying action mechanism still remains elusive [12].

Our previous study revealed QFXY composition with UPLC/Q-TOF-MS, consisting of 55 ingredients including 27 absorbable constituents [13]. In this study His\&Achinduced asthma model in guinea pigs was established, and QFXY was administered orally. HE stained sections were applied for QFXY effect evaluation. Customized microarrays and 2D electrophoresis (2DE) were adopted to detect differentially expressed genes and proteins ("diff genes" or "diff proteins" for short) respectively. Some diff proteins were identified with MALDI-TOF/MS. Cluster, GO and KEGG analyses enrich the functions and pathways of the diff genes and proteins. Based on asthma related genes from GAD and HPRD databases, the interaction network of all diff genes (including both diff genes from microarrays and diff genes blasted from diff proteins from 2DE) with asthma related genes was achieved, which indicated QFXY had multi-target regulation on asthma. Some detailed ingredients of QFXY may become candidate antiasthma drugs in the future.

\section{Methods}

\section{Drugs and animals}

QFXY pills (Lot. 100512) were provided by Tianjin Zhongxin Pharmaceutical Group. Guinea pigs of England specie, $(300 \pm 20) \mathrm{g}$, male and female, were purchased from Beijing Vital River Laboratory Animal Technology Co., Ltd. The animals were housed at $22 \pm$ $2^{\circ} \mathrm{C}$ with $55 \pm 10 \%$ humidity, $12 \mathrm{~h}$ light/dark cycle, and had free access to species-specific food and tap water. All experiments were carried out according to the Guide for the Care and Use of Experimental Animals. Studies were approved by the Institute Committee of the Animal Care of Nankai University, China.

\section{Protocol of asthma model}

Inside a container, guinea pigs were given the mixed solution of $0.1 \%$ histamine phosphate and $2 \%$ acetylcholine chloride for $10 \mathrm{~s}$ with ultrasonic sprayer $(0.5 \mathrm{~mL} / \mathrm{min})$. The time when asthma occurred was recorded [14]. The asthmatic guinea pigs were randomized into 3 groups, QFXY2 (397.5 mg/100g BW), QFXY1 (or "Q" for short) (132.5 mg/100g BW) and Model group ("M" for short) ( $\mathrm{n}=6$ for each group), were administrated orally with QFXY and normal saline respectively for 7 days. Again, guinea pigs were put into the glass cup and given $0.1 \%$ histamine phosphate for 10s, and prolonged period of asthma was recorded. There was another group without any treatment as the Normal group ("N" for short) for the following pathological sections and microarrays. The lung tissues of guinea pigs prepared for further experiments.

\section{Pathological analysis}

HE sections of bronchial and lung tissue of guinea pig were conducted according to the regular methods [15]. Briefly, the fresh lung tissue samples were fixed in 10\% formalin, and embedded in paraffin. Samples were cross-

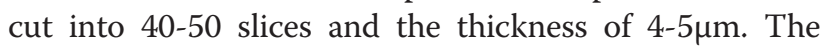
slices were stained by Hematoxylin-Eosin (HE). Finally, the stained sections were observed in light microscope (100x and 40x).

\section{Microarray procedures and data analysis}

Total RNA of 50mg lung tissues of each group was extracted [16] with Trizol (Invitrogen, USA), chloroform, isopropanol, 75\% ethanol, and purified using Nucleo Spin RNA Clean-up Kit (MN, Germany). RNA concentration and integrity were determined by UV-1800 Spectrophotometer (Shimadzu, 
Japan) and agarose gel electrophoresis. Four Guinea pigs gene expression chips were customized (Agilent Technologies, USA). The dual-channel chips were scanned with LuxScan 10KA dual channel laser scanner (CapitalBio, China). In the primary hybridization profiles, cy5 in red, cy3 in green, three chips were QFXY/ Normal, one chip was Model/Normal. The relative differential ratio of signal strength, (QFXY/Normal)/ (Model/Normal) presented the relatively varied gene expression of QFXY/Model. The relative ratios were for the following SAM (Significance Analysis of Microarrays) analysis for diff gene screening. 2_3, 2_4 and 2_9 were of the QFXY group. RNA of the QFXY group was isolated from each sample individually and was not pooled. But RNA samples from the Model group and Normal group were pooled to reduce biological differences [17]. SAM One Class method was adopted for the analysis of diff genes. Standard criteria for diff genes were $\mid$ Score $(\mathrm{d}) \mid \geq 2$ and Fold Change $\geq 2$ (or $\leq 0.5$ ). Cluster 3.0 was used with the hierarchical average linkage algorithm to get a heat map. In PubMed, the reference sequences of guinea pig were blasted to human genes, with the E value less than $1 \mathrm{e}-5$, and the similarity between two sequences spanned over half sequence length [18].
The human genes were imported Molecule Annotation System (MAS3.0) [19] for GO and Pathway analysis.

\section{D-electrophoresis and MS identification}

Proteins were isolated from $20 \mathrm{mg}$ lung tissues of each group with RIPA (Radio-Immunoprecipitation Assay) Lysis Buffer (SolarBio, China) containing 1Mm PMSF (Phenylmethanesulfonyl fluoride) for $15 \mathrm{~min}$ lysis on ice and centrifuged in $10000 \mathrm{~g}$. Protein concentration was diluted to $2 \mathrm{mg} / \mathrm{ml}$ by Bradford method [20]. In 2D-electrophoresis instrument (BIO-RAD), pH 3-10 precast IEF strips, $0.7 \mathrm{mg}$ sample loading, total $\mathrm{v} \cdot \mathrm{h} 80000,120 \mathrm{~g} / \mathrm{L}$ gel for SDSPAGE, and Coomassie brilliant blue staining method was adopted. The GS-800 scanner was used for acquiring image, with PDQuest 7.1 software for dot cutting, editing, detecting and matching. MS analysis providing purity, molecular weight, amino acid sequence, composition of peptide fragments, as well as the database support, differential proteins can be identified. Based on the MS report, protein score greater than 60 or single peptide score over 30 is more reliable. If more than one protein scored over 60 , the top ranked is more credible. C.I. \% over $95 \%$ is also reliable criterion. Besides, we also compared the theoretical protein molecular weight and isoelectric point with those we

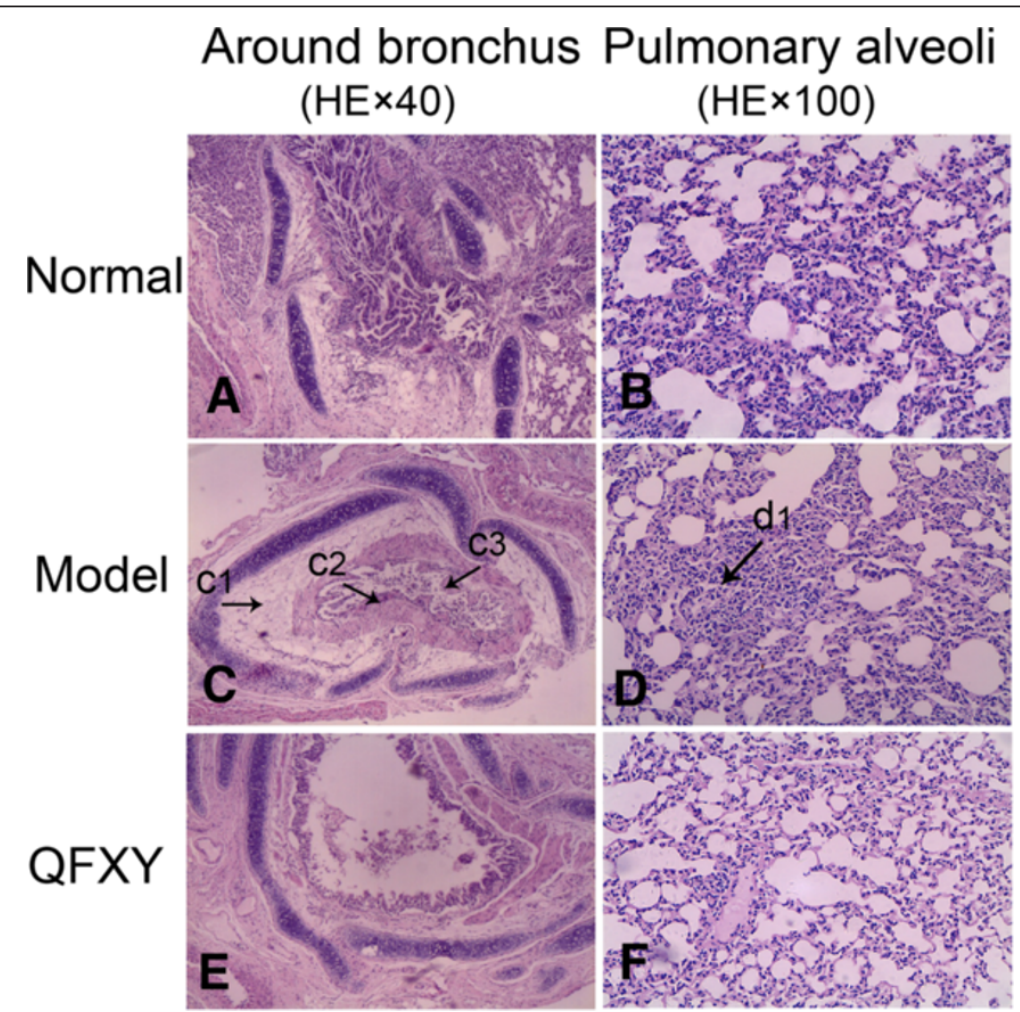

Figure 1 The pathological sections of each group (HEX40 and HEx100). (A\&B) Normal group, (C\&D) Model group, (E\&F) QFXY group. A, C and $\mathbf{E}$ showed lung tissues of guinea pigs around bronchus; B, D and $\mathbf{F}$ displayed around pulmonary alveoli. Comparing with the Normal group and QFXY group, obvious inflammatory cell infiltration in mucosa and submucosa seen in Model group. Arrow c1 displayed submucosa of spongiform vacuoles degeneration, necrosis; Arrow c2 displayed edema of tracheal mucosa, thickened bronchial walls, more goblet cells; Arrow c3 displayed narrowed or even blocked bronchial lumen, mucous plugs, necrosis mucosa; Arrow d1 displayed alveolar tissue consolidation. 
obtained in 2DE analysis. Furthermore, the diff proteins can be blasted into genes for further study.

\section{Quantitative real-time PCR and data analysis}

Validation of changes of diff genes in guinea pig lung tissues was carried out by real-time quantitative polymerase chain reaction (qPCR). First, total RNA was converted to cDNA using High Capacity cDNA Reverse Transcription Kits (ABI, USA). According to the reference sequences of guinea pig, the primers used are as follows: RHO (forward: 5'-CACCGCTCAACTACATCCTGC-3', Reverse: 5'- GCC CGAAGACGAAGTATCCA-3'), GNB1 (forward: 5'- TC CATCTACAATCTGAAGACTCGC -3', Reverse: 5' - TAGT CTGGTGTCGGGAGCG -3'), MAPK3 (forward: 5'- CTC AACCACATTCTGGGTATCC -3', Reverse: 5'- CCACCT TAGTCTTAGAGGGCAGA -3'), CLU (forward: 5'- GGA TGAAGGACCAGTGCGAG -3', Reverse: 5'- CGTGGAG ACATGGAGATAGGC -3'), ENO1 (forward: 5'- CAGAA GTCACAGCCAGCGTG -3', Reverse: 5' - CTTTGAGCAG CAGGCAGTTG -3'), HSP90 $\alpha$ (forward: 5'- GGCAGAG GCTGATAAGAACG -3', Reverse: 5'- AGTCATCCCTCA GCCAGAGA -3'), SERPINE2 (forward: 5'- CAGGGTCA GAAAACCTCCAT -3', Reverse: 5'- CTGCCCCATGAATA

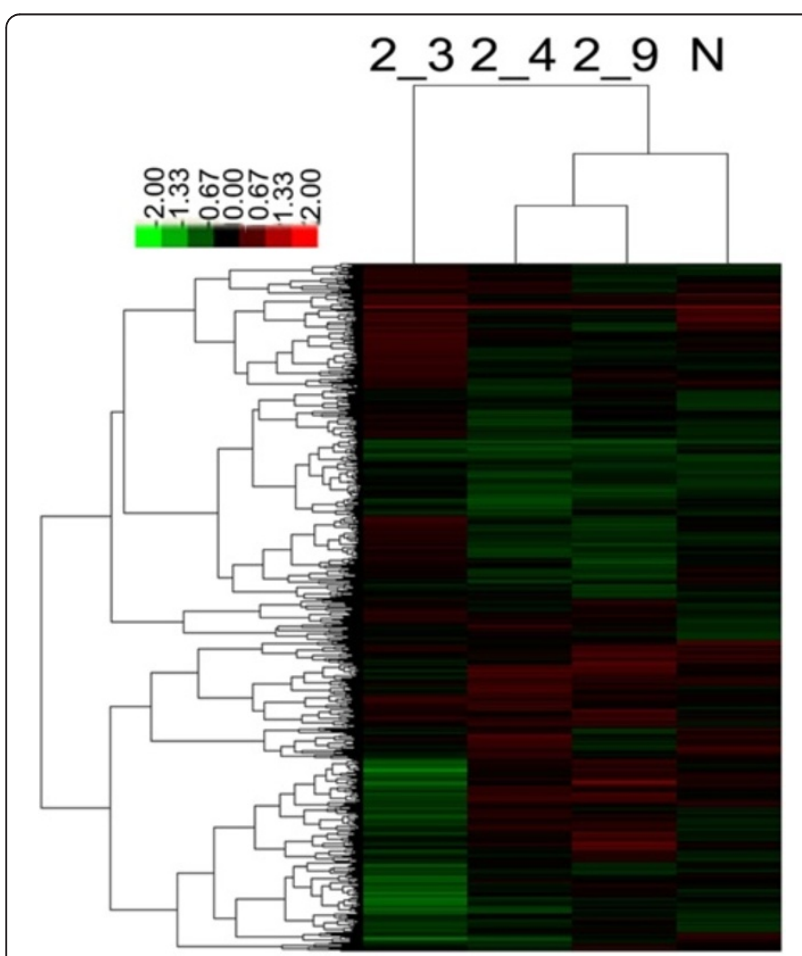

Figure 2 Heat map of the microarrays by hierarchical clustering. Heat map is a visual diagram, in which the vertical ordinates showed various chips, and the horizontal ordinates showed genes expressed in all chips. 2_3, 2_4, 2_9 were of the QFXY group. $N$ was the Normal group. Red indicated a minimum two fold increase in expression; green represented a minimum two fold decrease in expression, compared between the Normal control and QFXY treatments.
ACACAG -3'), GAPDH (forward: 5'- GAACATCATCC CCGCATCC -3', Reverse: 5'- GTCCTCGGTGTAGCCCA AGA -3'). Real-time PCR for quantitative assessment of mRNA expression was performed on LightCycler 2.0 (Roche, Swiss) with GoTaq qPCR Master Mix (Promega, USA) according to the manufacturer's protocol. The PCR conditions were as follows: $94^{\circ} \mathrm{C}$ for $2 \mathrm{~min}$, followed by 40 cycles of amplification $\left(95^{\circ} \mathrm{C}\right.$ for $15 \mathrm{~s}, 55^{\circ} \mathrm{C}$ for $30 \mathrm{~s}, 72^{\circ} \mathrm{C}$ for $30 \mathrm{~s}$ ), and a dissociation stage. $2^{-\Delta \Delta \mathrm{Ct}}$ method was applied for data analysis.

\section{Western blot of Hsp90}

The protein sample $(40 \mu \mathrm{g})$ was separated by $12 \%$ denaturing SDS-PAGE and blotted onto a nitrocellulose membrane. After electrophoresis, the proteins were transferred to nitrocellulose membrane by electrophoretic transfer system. The membranes were blocked in 5\% skimmed milk in TBS for $1 \mathrm{~h}$, and then incubated with primary antibody (anti-Hsp90 at 1:2000 dilution and anti- $\beta$-actin antibodies at 1:10000 dilution) (Wuhan Boster, China) overnight at $4^{\circ} \mathrm{C}$. The membranes were incubated for $2 \mathrm{~h}$ in horseradish peroxidase-conjugated goat anti-rabbit secondary antibody (1:2500 dilution) for $2 \mathrm{~h}$. Antigenantibody complex was visualized by enhanced chemiluminescence reagents Supersignal (Pierce, Rockford, IL). For quantification, Quantity One software (Bio Rad, USA) was used.

\section{QFXY-asthma target network construction}

Human protein interaction data were sourced from $\mathrm{Hu}-$ man Protein Reference Database (HPRD) [21] as the background. Asthma related genes from Genetic Association Database (GAD) [22] were annotated to the background network. Those nodes having direct interactions with asthma genes were used to build an asthma disease subnetwork. Keep the possibly same interactions in the subnetwork and HPRD network overlapped. By Cytoscape (Ver. 2.6.0) [23], the diff genes (from both microarray and $2 \mathrm{DE}$ data) were annotated in the asthma disease subnetwork and achieved QFXY-asthma target network.

\section{Statistics}

Data were presented as Mean \pm SD. The significance in mean values was analyzed by $t$ test for 2 groups and by analysis of variance (ANOVA) with least squares difference post-hoc test for more than 2 groups. Values were considered statistically different at $p<0.05$.

\section{Results}

\section{Histopathological results}

To test the QFXY effect, the pathological sections of lung tissues were stained by $\mathrm{HE}$ demonstrated in Figure 1. In the Model group (Figure 1C and Figure 1D), pathological sections showed significant edema of tracheal mucosa, 

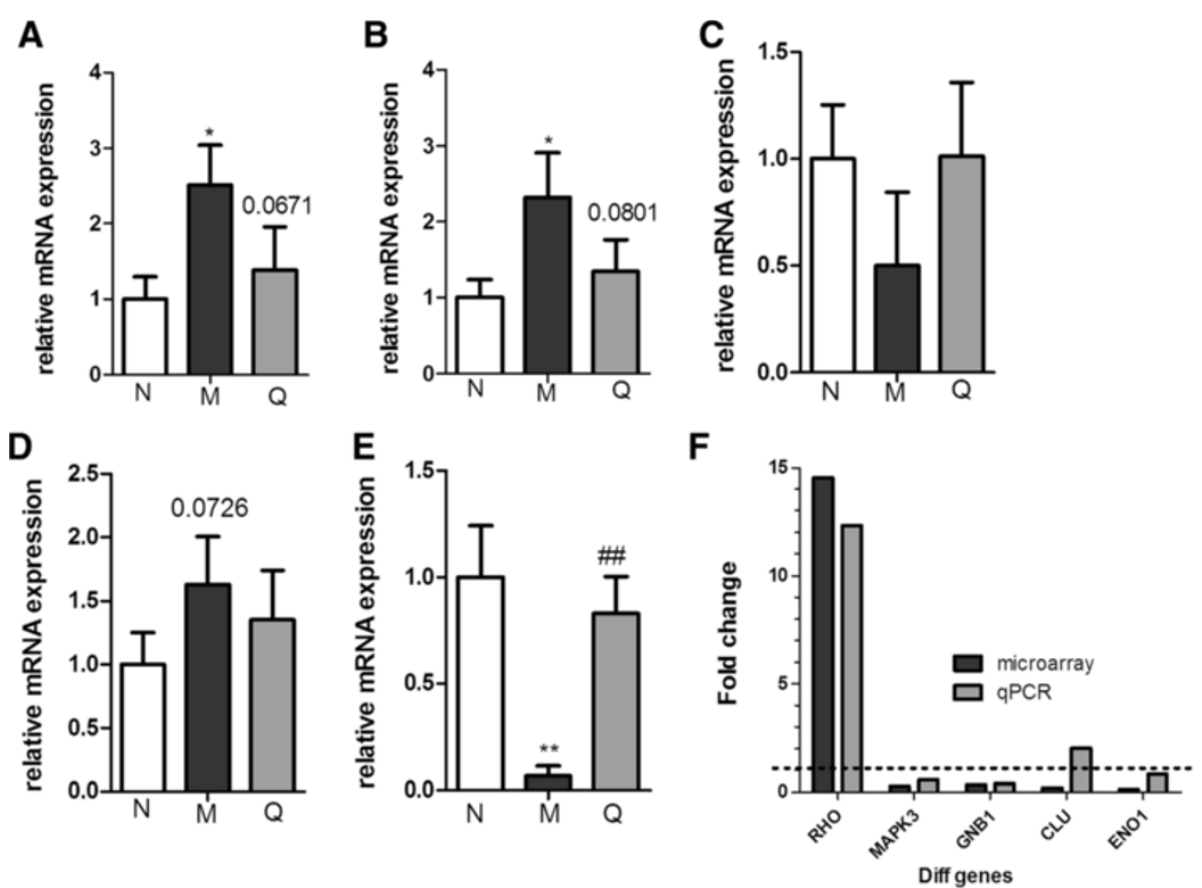

Figure 3 qPCR confirmation of the diff gene expressions with GAPDH as internal reference. Results were expressed as Mean \pm SD, $p<0.05$, ${ }^{* *} p<0.01$ vs. Control group; ${ }^{*} p<0.05,{ }^{\# \#} p<0.01$ vs. Model group.
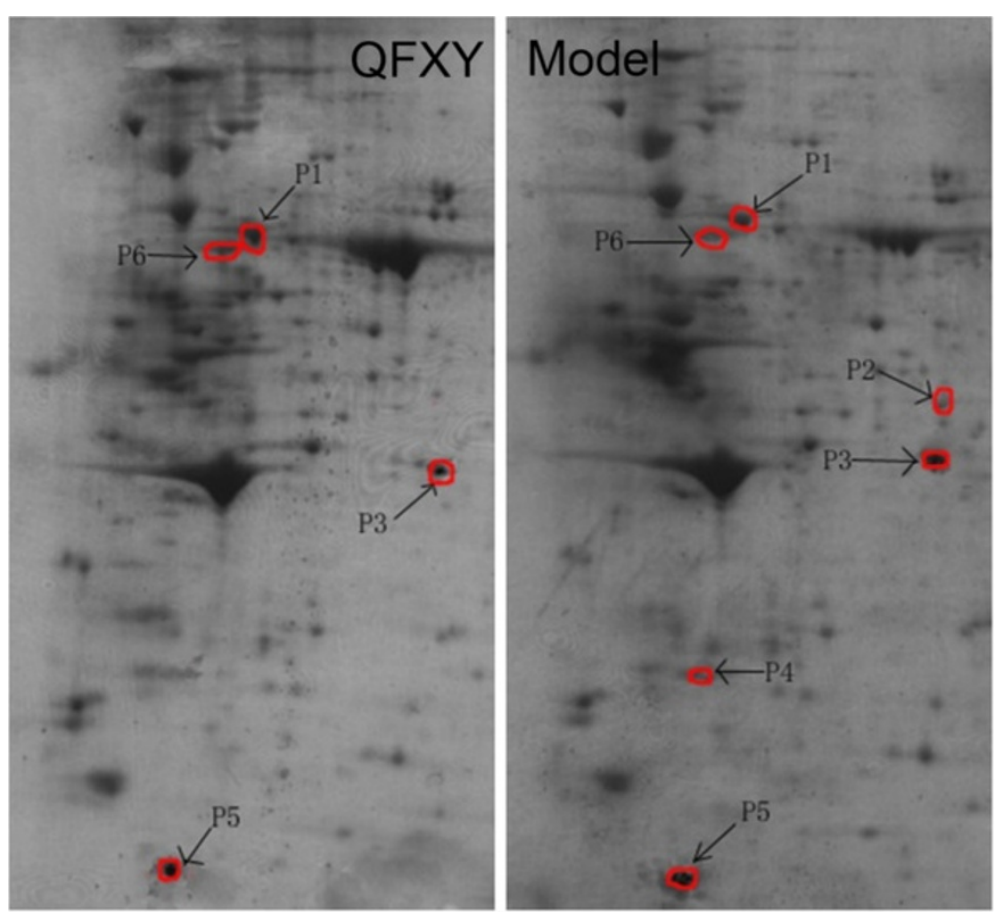

Figure 4 The 2DE results of QFXY and Model group. Identified diff proteins were marked by arrows in 2DE of QFXY group and Model group. 
Table 1 MS identification of some diff proteins

\begin{tabular}{|c|c|c|c|c|c|c|}
\hline \multirow{2}{*}{$\begin{array}{l}\text { Diff } \\
\text { proteins }\end{array}$} & \multirow{2}{*}{$\begin{array}{l}\text { Protein } \\
\text { score }\end{array}$} & \multirow{2}{*}{$\begin{array}{l}\text { Protein score } \\
\text { C. I. } \%\end{array}$} & \multicolumn{2}{|c|}{ Average $2 \mathrm{DE}$ data } & \multirow{2}{*}{$\begin{array}{l}\text { Diff ratio } \\
\text { (QFXY/Model) }\end{array}$} & \multirow[t]{2}{*}{ Human homolog } \\
\hline & & & QFXY & Model & & \\
\hline P1 & 946 & 100 & 55703.9 & 10498.7 & 5.31 & heat shock protein Hsp90-alpha isoform 2 \\
\hline P2 & 164 & 100 & $(-)$ & 2331.6 & & macrophage-capping protein \\
\hline P3 & 151 & 100 & 15264.5 & 25045.8 & 0.61 & microfibrillar-associated protein 4 \\
\hline P4 & 278 & 100 & $(-)$ & 3976.3 & & gamma-actin \\
\hline P5 & 348 & 100 & 40773.4 & 20867.1 & 1.95 & lamin-B1 \\
\hline P6 & 347 & 100 & 16005.9 & 2196.3 & 7.29 & serine proteinase inhibitor A3K \\
\hline
\end{tabular}

presenting mucosa epithelial cells swelling, some epithelial cells in spongiform vacuoles degeneration, necrosis and loss, and more goblet cells. Narrowed or even blocked bronchial lumen, thickened smooth muscles of the bronchial walls, and mucous plugs were visible and bronchial vascular congestion and angiogenesis, and inflammatory cell infiltration in mucosa and submucosa as well as perivascular tissues. In the Normal group (Figure 1A and Figure 1B), neither was obvious edema in airway mucosa, nor inflammatory cell infiltration in airway and vascular vessels. Bronchial tube cavity is smooth and unblocked. Comparing with the Model group, the QFXY group (Figure 1E and Figure 1F) has obvious change in bronchial

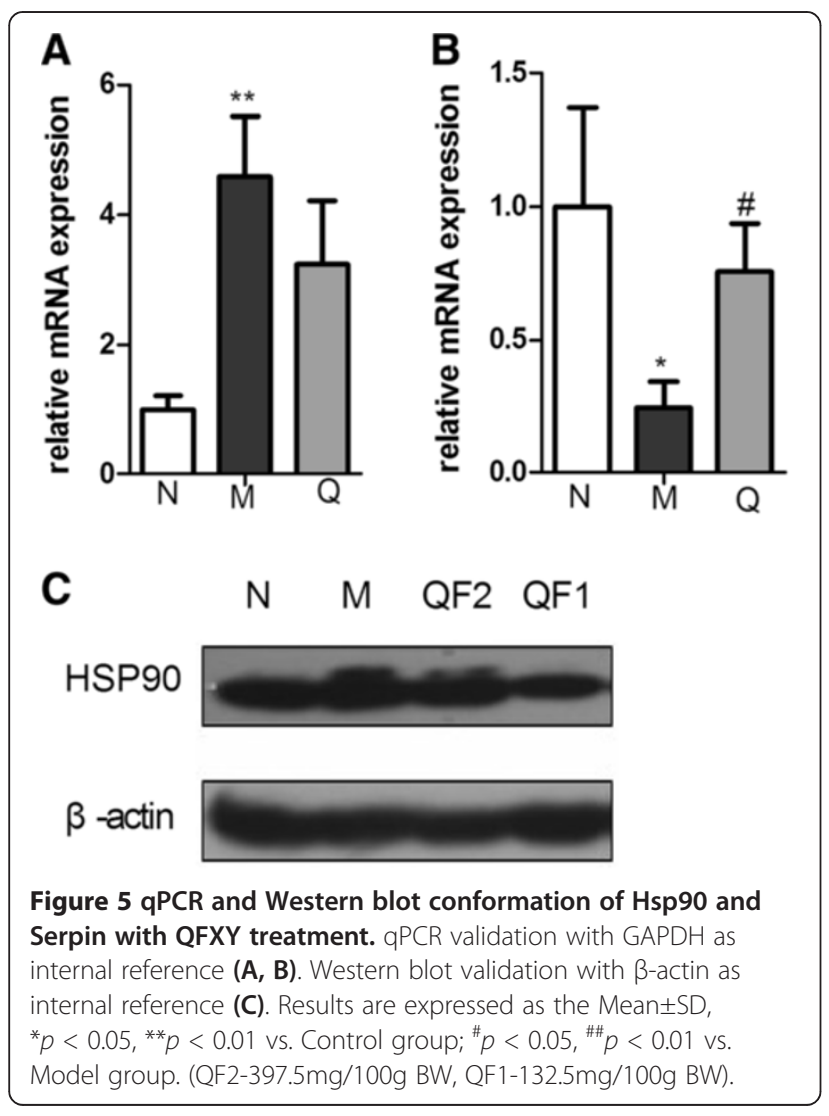

lung structure, more similar to the Normal group, which preliminarily showed sound effect.

\section{Microarray analysis and qPCR validation}

In our study, guinea pig cDNA microarrays were customized using the sequences as many as we could archive in NCBI EST database [24] (19911EST and 930CDS), which assemble can be used as a microarray design template for guinea pig. SAM analysis screened 55 diff genes of guinea pig, with 14 up-regulated and 41 down-regulated, see Additional file 1. Hierarchical Cluster analysis generated a heat map, shown in Figure 2, generally revealing gene expression module comparison of the samples. As shown in the Heat Map of the Figure 2, 2_4 and 2_9, the expression profile of the QFXY group had more similarity to that of the Normal group, which suggested that with the QFXY treatment, the overall gene expression profiles were inclined to the normal level, indicating the mitigation and improvement of asthma. The gene expression was verified with qPCR, seen in Figure 3A-3E. The correlation of expression level in microarray and qPCR seen in Figure 3F.

\section{DE, MS identification and validation}

$2 \mathrm{DE}$ results were seen in Figure 4. Some diff proteins were identified using MALDI-TOF/MS seen in Table 1. Due to limited research data of guinea pig, diff proteins were blasted into human proteins as well as relevant genes. Protein expression was validated with qPCR and Western blot displayed in Figure 5. The expression level of Hsp90 decreased and Serpin increased with QFXY treatment comparing with the Model group.

\section{GO and pathway enrichments}

There are few guinea pig research data of definite functions of genes and signal pathways. In NCBI, we blasted 55 diff genes of guinea pig and got 27 human homologues, see Additional file 2. The molecular function, biological process and cellular component of the 27 diff genes see Additional file 3, especially involved in such biological processes as signal transduction, protein phosphorylation, stress response and etc. The diff genes participate in some pathways, see Additional file 4. Sourced from KEGG 


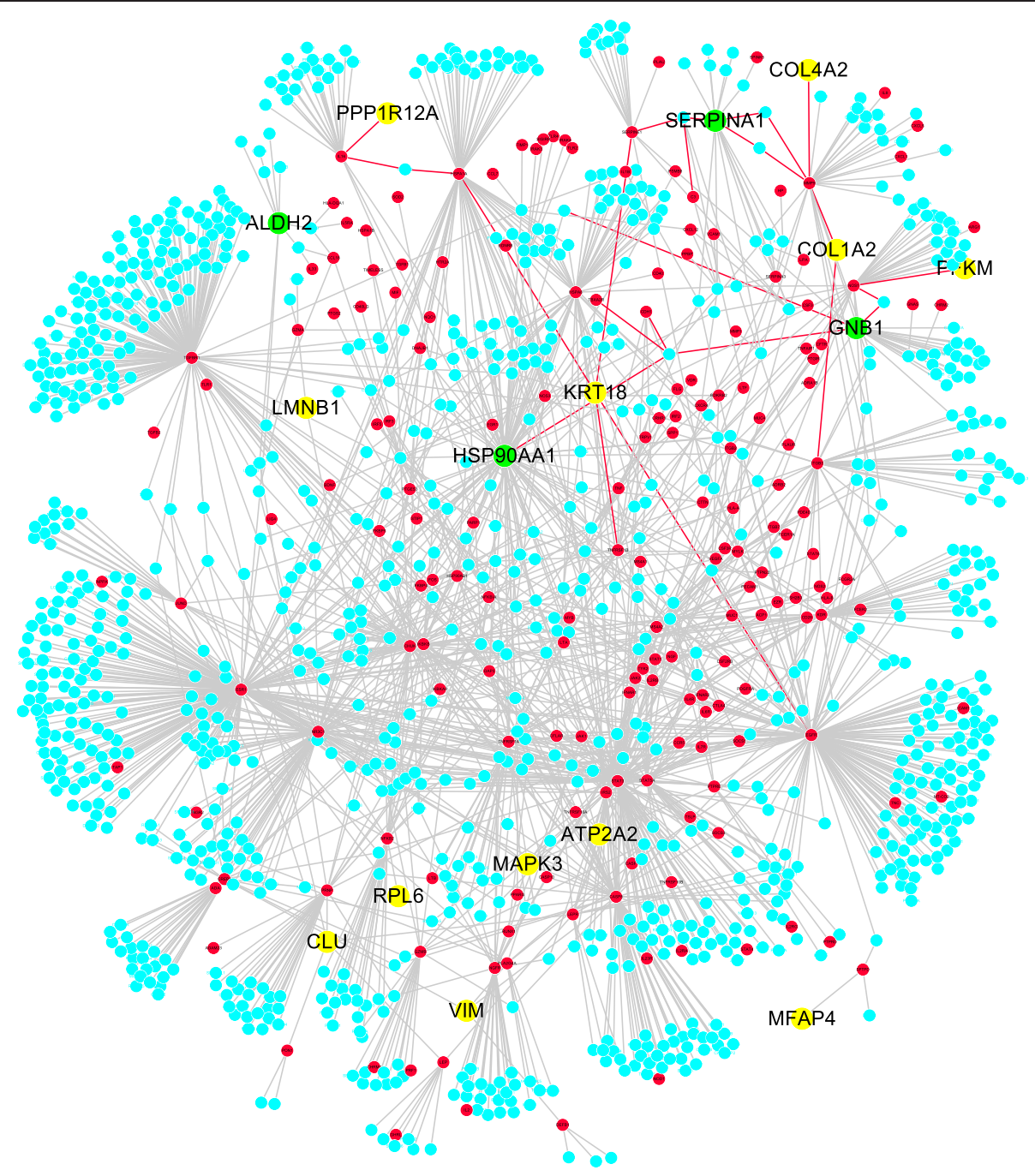

Figure 6 QFXY-asthma target network based on interactions between all diff genes and asthma related genes. Red for known asthma genes (resourced from HRPD\&GAD), green for diff genes (including those blasted from diff proteins), yellow for diff genes which had direct interactions with asthma genes, blue for other genes directly interacting with asthma genes.

[25], GenMapp [26] and BioCarta [27], diff genes participated in several common signal pathways, some of which were involved in inflammation (Hs_TNFalpha-NF- $\kappa$ B_NetPath_9, Prostate cancer pathway), cell movement and proliferation as well as airway remodelling of the cytoskeleton and extracellular matrix (Hs_Regulation_of_Actin_Cytoskeleton, extracellular matrix, intermediate filament cytoskeleton, structural constituent of cytoskeleton, actin binding, actin cytoskeleton), multi-level signaling protein folding (protein folding, unfolded protein binding), cell adhesion and signal transduction (Adherens junction, Focal adhesion), and so on. Important genes involved include HSP90A1, SERPINA1, MAPK3, ACTG1, VIM, TNNT2, GNB1, CRYAA, CRYAB, COL4A2, COL1A2 and so on. The compiled file and detailed pathways see Additional file 5 .

\section{QFXY-asthma target network}

A network containing 1214 nods and 1886 interactions was constructed as QFXY-asthma target network. In Figure 6, red for known asthma genes (sourced from HRPD\&GAD), green for diff genes (including those blasted from diff proteins), yellow for diff genes which had direct interactions with asthma genes, blue for other genes directly interacting with asthma genes. In total, the network contained 16 diff genes, 182 asthma genes (from databases), and 1016 genes directly interacting with asthma genes.

\section{Discussion}

Studies in animal models form the basis for our current understanding of the pathophysiology of asthma, and are central to the preclinical development of drug therapies 
[28]. Guinea pigs have been the most commonly used small animal species in preclinical studies related to asthma and COPD [29]. $\beta_{2}$-adenoceptor agonists and antimuscarinic drugs prevent antigen-induced bronchoconstriction in actively sensitized guinea pigs in a dosedependent manner. Histamine is the major mediator in guinea pigs but not in humans [30]. Asthma is a complex disease defined by reversible airway narrowing, acute and chronic airway inflammation, airway hyperresponsiveness (AHR) and airway tissue remodelling, in which accumulation of airway smooth muscle (ASM) is a prominent and widely reported feature [31]. In the pharmacodynamics study, the prolonged asthma time [12] and HE sections showed that QFXY had significant effects on asthma, reducing edema in airway mucosa and inflammatory cell infiltration in airway and vascular vessels. They were also beneficial to reducing airway remodelling.

Among up-regulated genes, the fold change of RHO almost ranked top (Additional file 2). Among downregulated genes, CLU and ENO1 had greater changes. Among 2D results, fold changes of Hsp90 and Serpin were of greater change (Table 1). Besides, references and literatures about every diff molecules were retrieved, of which some were related to the process of inflammation or asthma or lung diseases, such as GNB1 [32,33], MAPK3 [34-37]. Altogether, with the combined consideration of fold changes and references, these genes (RHO, CLU, ENO1, GNB1, MAPK3) and proteins (Hsp90, Serpin) were selected for validation test.

The GO annotation suggested that QFXY might influence the inflammation, signal transduction, stress response, the apoptosis of endothelial and bronchial cells. Pathway analysis revealed that different genes were involved in the signaling pathways, including focal adhesion pathway, cell-extracellular matrix interactions pathway, TGF-beta signaling pathways, NK cell-me diated cytotoxic pathway and so on, which are all related with cell signaling, inflammation, mast cells and NK cells. Many asthma drugs also participated in those pathways in variety of mechanisms, targeting kinases, receptors or related proteins, affecting inflammation response, mitosis, angiogenesis, apoptosis, and anti-oxidation, to play a role in asthma. The qPCR change profile was basically in line with the microarray results, proving the reliability of microarray data. The commonly shared signal pathways of diff genes and diff proteins combined the genomics and proteomics together, to manifest the underlying mechanism of QFXY effects.

The Mapk3/Erk signaling cascade is a central Mapk pathway that plays a role in the regulation of various cellular processes such as proliferation, differentiation, development, and inflammation reactions and etc. [34-37]. Inhibition of this kinase strongly decreased the expression of pro-inflammatory genes encoding growthregulated proteins (Gro-a, Gro-b and Gro-g) and interleukins (IL-1 $\beta$, IL-6 and IL-8) [36]. Mapk can participate in the regulation of $\mathrm{NF}-\mathrm{kB}$ transcriptional activity $[38,39]$. Our previous study also presented decreasing erk expression and NF- $\mathrm{kB}$ inhibition [13].

Hsp90, as a molecular chaperone, has interactions with proteins, such as Akt and Raf-1 [40]. Akt is a downstream effecter molecule of phosphoinositide 3-kinase and is thought to mediate many immune and inflammatory responses. It is also involved in the activation of NF-kB [41]. Amino acid residues 229-309 of Akt were involved in the binding to Hsp90 and amino acid residues 327-340 of Hsp90 $\beta$ were involved in the binding to Akt [42]. Hsp90 plays an important role in maintaining Akt kinase activity. In our study, 2D and Western blot showed decreased Hsp90 after QFXY treatment, as well as less NF- $\mathrm{kB}$ activity [13], indicating QFXY may affect the binding of Hsp90 and Akt, which needs further confirmation.

GTP-binding protein beta1 subunit gene (GNB1), its up-regulation appears to be one of the candidate processes of sensitization. It also has $N F-k B$ recognition sites [32]. The Ectodysplasin is involved in binding to its ligand EDA-A1 and activates the NF- $\mathrm{B}$ intracellular signaling pathway by interaction through its death domain with the adaptor protein EDARADD [33]. Down-regulated GNB1 and EDARADD gene expression decreased NF- $\kappa$ B activity for anti-inflammation.

Serpins form an enormous superfamily of 40-60-kDa proteins found in almost all types of organisms. Most have evolved to finely regulate complex proteolytic pathways, such as blood coagulation, fibrinolysis, and inflammation $[43,44]$. $\alpha 1$-antitrypsin (AAT) is an archetype member of the serpin supergene family. The reduced serum levels of AAT contribute to the development of chronic obstructive pulmonary disease (COPD). In addition to protease inhibition, AAT shows anti-in flammatory, immunomodulatory and antimicrobial properties $[45,46]$. SerpinA1 is an endogenous antiinflammatory factor, and its anti-inflammatory effects may be mediated through antioxidant activity [47]. Compared with the Model group, the HE sections of the QFXY group showed less inflammation and mucosa hyperplasia, and the $2 \mathrm{D}$ and $\mathrm{qPCR}$ proved higher SerpinA1 expression, which indicating specific ingredients in QFXY can activate SerpinA1.

Asthma is a disease characterized by persistent inflammation and structural changes in the airways referred to as airway remodelling., including smooth muscle hypertrophy, goblet cell hyperplasia, subepithelial fibrosis, and angiogenesis. Vascular remodelling in asthmatic lungs results from increased angiogenesis, mediated by vascular endothelial growth factor (VEGF). In addition, 
VEGF induces allergic inflammation, enhances allergic sensitization, and has a role in Th2 type inflammatory responses [48]. Matrix GLA protein (MGP) has a role in endothelial cell (EC) function [49]. MGP modulates the activity of transforming growth factor (TGF) $\beta$ superfamily, which is critical for morphogenesis and development [50]. MGP can stimulate VEGF expression through increased TGF- $\beta$ activity in endothelial cells [51]. Comparing with the Model group, HE sections in the QFXY group showed less pulmonary consolidation, which means QFXY help alleviate lung tissue remodelling.

Asthma is featured by reversible airway obstruction. The lack of full reversibility in some asthmatic patients may be due to chronic airway remodelling [52]. It appears that inflammation and remodelling are interdependent processes that clearly influence the clinical long term evolution of asthma [53]. The ECM can act as a reservoir for an increasing number of growth factors. These growth factors can be rapidly released from the ECM to allow extracellular signaling regulated by the growth factors to proceed without the need for new protein synthesis [54].

In QFXY-asthma target network, Hsp90 $\alpha$, Mapk3, VIM were hub proteins suggesting that they may be some targets of QFXY pills. The complicated interaction network suggested that QFXY pills affected a complex system regulating inflammation and immune reactions. Seen from the above complex network, QFXY interacts with asthma related genes in both direct and indirect way, affecting several signal pathways. In the previous study, 55 ingredients have been identified, including 27 absorbable constituents in QFXY, among which there are 19 ingredients affect inflammatory pathways, typically they are sulfur containing alkynes, such as arctic acid; lignans, such as arctigenin; phenolic acids, such as sinapic acid; steroids, such as cholic acid [13]. In the following study, other effects of these ingredients, such as alleviating airway hyperresponsiveness (AHR) and airway tissue remodelling will be further explored.

\section{Conclusions}

A primarily combined genomic and proteomic screen of QFXY targets displayed a series of candidate genes and proteins, which indicated that the effect of QFXY relied on combined mechanism, anti-inflammation and anti-remodelling, as well as influence signal transduction in vivo.

\section{Additional files}

Additional file 1: List of diff genes of microarrays.xls

Additional file 2: Blasting list of diff genes.xls

Additional file 3: $\mathrm{GO}$ analysis of diff genes.xls
Additional file 4: KEGG pathways analysis of diff genes.xls

Additional file 5: List of commonly shared pathway (sourced from KEGG, GenMAPP, and BioCarta) by diff genes and diff proteins.doc

\section{Competing interests}

The authors declare that they have no competing interests.

\section{Authors' contributions}

ZZ, GB and HW contributed to the conception and design of the study. ZZ completed the experiment. YM assisted the bioinformatics, PP finished 2D-MS, BC assisted the animal models. ZZ and HW drafted the manuscript. All authors contributed to further writing of the manuscript. All authors read and approved of the final manuscript.

\section{Acknowledgements}

This study was financially supported by grants from the National Nature Science Fund (NNSF) of China (No. 81173638). Thanks to Professor Gu'an Luo, Associate Professor Yuanyuan Hou and Lecturer Min Jiang and Chunfeng Xie, for their sincere instructions and help. Also thanks to Professor Xiaobo Tang and researcher Chuanfei Yu and Shan Yun for their advice on English writing.

\section{Author details}

${ }^{1}$ College of Pharmacy, Nankai University, Tianjin, People's Republic of China. ²Department of Pharmacy, Tianjin People's Hospital, Tianjin, People's Republic of China. ${ }^{3}$ Department of Pharmacology, Tianjin Medical University, Tianjin, People's Republic of China.

Received: 25 March 2013 Accepted: 2 August 2013

Published: 6 August 2013

\section{References}

1. Bateman ED, Hurd SS, Barnes PJ, Bousquet J, Drazen JM, FitzGerald M, Gibson P, Ohta K, O'Byrne P, Pedersen SE, Pizzichini E, Sullivan SD, Wenzel $\mathrm{SE}, \mathrm{Zar} \mathrm{HJ}$ : Global strategy for asthma management and prevention: GINA executive summary. Eur Respir J 2008, 31(1):143-178.

2. Holgate ST, Davies DE: Rethinking the pathogenesis of asthma. Immunity 2009, 31(3):362-367.

3. Gern JE, Lemanske RF, Busse WW: Early life origins of asthma. J Clin Invest 1999, 104(7):837-843.

4. Claudio L, Tulton L, Doucette J, Landrigan PJ: Socioeconomic factors and asthma hospitalization rates in New York City. J Asthma 1999, 36(4):327-343.

5. Bousquet J, Jeffery PK, Busse WW, Johnson M, Vignola AM: Asthma From bronchoconstriction to airways inflammation and remodeling. Am J Respir Crit Care Med 2000, 161(5):1720-1745.

6. Kroegel C: Global Initiative for Asthma (GINA) guidelines: 15 years of application. Expert Rev Clin Immunol 2009, 5(3):239-249.

7. Dorinsky: The salmeterol multicenter asthma research trial: A comparison of usual pharmacotherapy for asthma or usual pharmacotherapy plus salmeterol (vol 129, pg 15, 2006). Chest 2006, 129(5):1393.

8. Gibson PG, Powell H, Ducharme FM: Differential effects of maintenance long-acting beta-agonist and inhaled corticosteroid on asthma control and asthma exacerbations. J Allergy Clin Immun 2007, 119(2):344-350.

9. Lemanske RF, Sorkness CA, Mauger EA, Lazarus SC, Boushey HA, Fahy JV, Drazen JM, Chinchilli VM, Craig T, Fish JE, Ford JG, Israel E, Kraft M, Martin RJ, Nachman SA, Peters SP, Spahn JD, Szefler SJ: Inhaled corticosteroid reduction and elimination in patients with persistent asthma receiving salmeterol - A randomized controlled trial. Jama-J Am Med Assoc 2001, 285(20):2594-2603.

10. Wechsler ME, Pauwels R, Drazen JM: Leukotriene modifiers and ChurgStrauss syndrome - Adverse effect or response to corticosteroid withdrawal? Drug Saf 1999, 21(4):241-251.

11. Barnes PJ: Theophylline - New perspectives for an old drug. Am J Resp Crit Care 2003, 167(6):813-818.

12. Hou Y, Li R, Shi Q, Cheng B, Wang L, Bai R: Investigation of the Antiasthmatic Mechanism of QingFeiXiaoYanWan. Acta Scientiarum Naturalium Universitatis Nankaiensis 2009, 5:103-107.

13. Cheng B, Hou Y, Wang L, Dong L, Peng J, Bai G: Dual-bioactivity-based liquid chromatography-coupled quadrupole time-of-flight mass 
spectrometry for NF-kappaB inhibitors and beta2AR agonists identification in Chinese Medicinal Preparation Qingfei Xiaoyan Wan. Anal Bioanal Chem 2012, 404(8):2445-2452.

14. Vogel G, Vogel WH, Vogel HG: Drug Discovery and Evaluation: Pharmacological Assays. Berlin: Springer; 1997.

15. Fan X, Shi M, Wang Y, Liang Q, Luo G: Transcriptional profiling analysis of HMP-treated rats with experimentally induced myocardial infarction. J Ethnopharmacol 2011, 137(1):199-204.

16. Mutter $\mathrm{GL}$, Zahrieh $\mathrm{D}$, Liu CM, Neuberg D, Finkelstein D, Baker HE, Warrington JA: Comparison of frozen and RNALater solid tissue storage methods for use in RNA expression microarrays. BMC Genomics 2004, 5:88-94.

17. Pedotti P, Hoen PA T, Vreugdenhil E, Schenk GJ, Vossen RH, Ariyurek Y, De Hollander M, Kuiper R, Van Ommen G, Den Dunnen JT, Boer JM, De Menezes RX: Can subtle changes in gene expression be consistently detected with different microarray platforms? BMC Genomics 2008, 9:124-136.

18. Basic BLAST. http://blast.ncbi.nlm.nih.gov/Blast.cgi.

19. Molecule Annotation System. http://bioinfo.capitalbio.com/mas3/.

20. Fan X, Li X, Lv S, Wang Y, Zhao Y, Luo G: Comparative proteomics research on rat MSCs differentiation induced by Shuanglong Formula. J Ethnopharmacol 2010, 131(3):575-580.

21. Human Protein Reference Database. http://www.hprd.org/.

22. Genetic Association Database. http://geneticassociationdb.nih.gov/.

23. Cline MS, Smoot M, Cerami E, Kuchinsky A, Landys N, Workman C, Christmas R, Avila-Campilo I, Creech M, Gross B, Hanspers K, Isserlin R, Kelley R, Killcoyne S, Lotia S, Maere S, Morris J, Ono K, Pavlovic V, Pico AR, Vailaya A, Wang PL, Adler A, Conklin BR, Hood L, Kuiper M, Sander C, Schmulevich I, Schwikowski B, Warner GJ, et al: Integration of biological networks and gene expression data using Cytoscape. Nat Protoc 2007, 2(10):2366-2382

24. NCBI EST database. http://www.ncbi.nlm.nih.gov/nucest/.

25. KEGG PATHWAY Database. http://www.genome.jp/kegg/pathway.html.

26. GenMapp: Gene Map Annotation and Pathway Profiler. http://www.genmapp. org/default.html.

27. BioCarta. http://www.biocarta.com/genes/index.asp.

28. Bates JH, Rincon M, Irvin CG: Animal models of asthma. Am J Physiol Lung Cell Mol Physiol 2009, 297(3):L401-L410.

29. Canning BJ: Modeling asthma and COPD in animals: a pointless exercise? Curr Opin Pharmacol 2003, 3(3):244-250.

30. Szelenyi I: Animal models of bronchial asthma. Inflamm Res 2000, 49(12):639-654.

31. Holgate ST: Pathogenesis of asthma. Clin Exp Allergy 2008, 38(6):872-897.

32. Kitanaka J, Kitanaka N, Takemura M, Wang XB, Hembree CM, Goodman NL, Uhl GR: Isolation and Sequencing of a putative promoter region of the murine G protein beta 1 subunit (GNB1) gene. Dna Sequence 2002, 13(1):39-45

33. Moya-Quiles MR, Ballesta-Martinez MJ, Lopez-Gonzalez V, Glover G, Guillen-Navarro E: A compound heterozygous mutation in the EDAR gene in a Spanish family with autosomal recessive hypohidrotic ectodermal dysplasia. Arch Dermatol Res 2010, 302(4):307-310.

34. Shaul YD, Seger R: The MEK/ERK cascade: From signaling specificity to diverse functions. Bba-Mol Cell Res 2007, 1773(8):1213-1226.

35. McCubrey JA, Milella M, Tafuri A, Martelli AM, Lunghi P, Bonati A, Cervello M, Lee JT, Steelman LS: Targeting the Raf/MEK/ERK pathway with smallmolecule inhibitors. Curr Opin Investig Drugs 2008, 9(6):614-630.

36. Verhaeghe C, Remouchamps C, Hennuy B, Vanderplasschen A, Chariot A Tabruyn SP, Oury C, Bours V: Role of IKK and ERK pathways in intrinsic inflammation of cystic fibrosis airways. Biochem Pharmacol 2007, 73(12):1982-1994.

37. Perkins ND: Post-translational modifications regulating the activity and function of the nuclear factor kappa B pathway. Oncogene 2006, 25(51):6717-6730.

38. Weiss T, Shalit I, Blau H, Werber S, Halperin D, Levitov A, Fabian I: Anti-inflammatory effects of moxifloxacin on activated human monocytic cells: Inhibition of NF-KB and mitogen-activated protein kinase activation and of synthesis of proinflammatory ctokines. Antimicrob Agents Ch 2004, 48(6):1974-1982.

39. Lee FS, Peters RT, Dang LC, Maniatis T: MEKK1 activates both I kappa B kinase alpha and I kappa B kinase beta. P Natl Acad Sci Usa 1998, 95(16):9319-9324.

40. Fontana J, Fulton D, Chen Y, Fairchild TA, McCabe TJ, Fujita N, Tsuruo T, Sessa WC: Domain mapping studies reveal that the M domain of hsp90 serves as a molecular scaffold to regulate Akt-dependent phosphorylation of endothelial nitric oxide synthase and NO release. Circ Res 2002, 90(8):866-873.

41. Ozes ON, Mayo LD, Gustin JA, Pfeffer SR, Pfeffer LM, Donner DB: NF-kappa $B$ activation by tumour necrosis factor requires the Akt serine-threonine kinase. Nature 1999, 401(6748):82-85.

42. Sato S, Fujita N, Tsuruo T: Modulation of Akt kinase activity by binding to Hsp90. P Natl Acad Sci Usa 2000, 97(20):10832-10837.

43. Gettins P, Olson ST: Exosite Determinants of Serpin Specificity. J Biol Chem 2009, 284(31):20441-20445.

44. Ekeowa UI, Marciniak SJ, Lomas DA: alpha(1)-antitrypsin deficiency and inflammation. Expert Rev Clin Immunol 2011, 7(2):243-252.

45. Janciauskiene SM, Bals R, Koczulla R, Vogelmeier C, Kohnlein T, Welte T: The discovery of alpha 1-antitrypsin and its rote in health and disease. Resp Med 2011, 105(8):1129-1139.

46. Shigetomi H, Onogi A, Kajiwara H, Yoshida S, Furukawa N, Haruta S, Tanase Y, Kanayama S, Noguchi T, Yamada Y, Oi H, Kobayashi H: Anti-inflammatory actions of serine protease inhibitors containing the Kunitz domain. Inflamm Res 2010, 59(9):679-687.

47. Zhang B, Hu Y, Ma JX: Anti-inflammatory and antioxidant effects of SERPINA3K in the retina. Invest Ophthalmol Vis Sci 2009, 50(8):3943-3952.

48. Meyer N, Akdis CA: Vascular Endothelial Growth Factor as a Key Inducer of Angiogenesis in the Asthmatic Airways. Curr Allergy Asthma Rep 2013, 13(1):1-9.

49. Budd DC, Holmes AM: Targeting TGF beta superfamily ligand accessory proteins as novel therapeutics for chronic lung disorders. Pharmacol Therapeut 2012, 135(3):279-291.

50. Engelse MA, Neele JA, Bronckers A, Pannekoek H, De Vries C: Vascular calcification: expression patterns of the osteoblast-specific gene core binding factor alpha- 1 and the protective factor matrix gla protein in human atherogenesis. Cardiovasc Res 2001, 52(2):281-289.

51. Bostrom K, Zebboudj AF, Yao YC, Lin TS, Torres A: Matrix GLA protein stimulates VEGF expression through increased transforming growth factor-beta 1 activity in endothelial cells. J Biol Chem 2004, 279(51):52904-52913.

52. Boulet LP, Turcotte H, Laviolette M, Naud F, Bernier MC, Martel S, Chakir J: Airway hyperresponsiveness, inflammation, and subepithelial collagen deposition in recently diagnosed versus long-standing mild asthma - Influence of inhaled corticosteroids. Am J Resp Crit Care 2000, 162(4):1308-1313.

53. Vignola AM, Mirabella F, Costanzo G, Di Giorgi R, Gjomarkaj M, Bellia V Bonsignore G: Airway remodeling in asthma. Chest 2003, 123S(3):417S-422S

54. Burgess JK: The role of the extracellular matrix and specific growth factors in the regulation of inflammation and remodelling in asthma. Pharmacol Therapeut 2009, 122(1):19-29.

doi:10.1186/1472-6882-13-206

Cite this article as: Zhao et al:: Qingfei Xiaoyan Wan alleviates asthma through multi-target network regulation. BMC Complementary and Alternative Medicine 2013 13:206.

\section{Submit your next manuscript to BioMed Central and take full advantage of:}

- Convenient online submission

- Thorough peer review

- No space constraints or color figure charges

- Immediate publication on acceptance

- Inclusion in PubMed, CAS, Scopus and Google Scholar

- Research which is freely available for redistribution

Submit your manuscript at www.biomedcentral.com/submit
C Biomed Central 